\title{
Method for spectral phase retrieval of single attosecond pulses utilizing the autocorrelation of photoelectron streaking spectra
}

\author{
Wei-Wei Yu, ${ }^{1,2}$ Xi Zhao, ${ }^{2}$ Hui Wei, ${ }^{2}$ Su-Ju Wang, ${ }^{2}$ and C. D. Lin ${ }^{2}$ \\ ${ }^{1}$ School of Physics and Electronic Technology, Liaoning Normal University, Dalian 116029, People's Republic of China \\ ${ }^{2}$ Department of Physics, Kansas State University, Manhattan, Kansas 66506, USA
}

(Received 14 September 2018; revised manuscript received 31 December 2018; published 4 March 2019)

\begin{abstract}
We propose an algorithm for retrieving the spectral phase of an isolated attosecond pulse based on the photoelectron spectra generated in atoms by the attosecond pulse in the presence of a time-delayed infrared laser. Instead of employing the whole set of the streaking spectra as in the previous phase retrieval algorithms, we calculate the autocorrelation $(\mathrm{AC})$ of the streaking trace, and use it to extract the spectral phase. We illustrate that this method can be used to extract narrow- as well as broadband attosecond pulses. The method converges much faster and is more accurate. We also define two parameters, $V$ and $A$. By comparing the AC pattern and the $V$ and $A$ parameters from the experiment and from the retrieved pulse, this method provides a metric to evaluate the accuracy of the retrieved pulse.
\end{abstract}

DOI: 10.1103/PhysRevA.99.033403

\section{INTRODUCTION}

With the advent of attosecond pulse trains (APTs) [1] and isolated attosecond pulses (IAPs) [2] since 2001, attosecond pulses have been used to study electron dynamics in pumpprobe experiments [3]. In particular, with various generation techniques, IAPs with ever shorter durations, for example, pulses of duration as short as 130 as [4], 80 as [5], 67 as [6], and then 53 as [7] and 43 as [8] have been reported. To obtain the pulse duration, whether an APT or an IAP, photoelectron spectra (called spectrogram or streaking trace) generated by the attosecond pulse in the presence of an infrared or a mid-infrared laser are measured by varying the time delay between the two pulses. The spectral range of the attosecond pulses covers from the extreme ultraviolet (XUV) to soft $\mathrm{x}$ rays (SXRs). The polarizations of the two pulses are chosen to be parallel and photoelectron spectra are measured in the same direction. To find out the pulse characteristic in the time domain, both the spectral amplitude and phase of the pulse have to be determined. Using atomic targets, the spectral amplitude of an IAP can be easily calculated from the photoelectron spectra generated by the attosecond pulses alone since atomic photoionization cross sections of atoms have long been accurately determined from measurements, or from accurate theoretical calculations. To obtain the spectral phase of an APT, the so-called RABITT (reconstruction of attosecond beating by interference of two-photon transitions) method [9] is commonly used, while for an IAP, the spectral phase is commonly extracted using the FROG-CRAB (frequency-resolved optical gating for complete reconstruction of attosecond bursts) method [10]. Both methods are based on some approximate theory.

In this article we will focus on the phase retrieval of an IAP. Once the spectral amplitude and phase are obtained, its Fourier transform would give the temporal amplitude and phase of the IAP. For an attosecond pulse, one would generally show the retrieved spectral phase and the intensity in the time domain. The pulse duration is an approximate characterization of a pulse. We will focus on the accurate retrieval of the spectral phase, not just the pulse duration.

The accuracy of spectral phase retrieval is limited by a number of factors. To begin with, one needs a simple and accurate theory that can describe the photoelectron spectra or the streaking trace. The commonly used strong-field approximation (SFA) is simple, but it is not accurate for low-energy photoelectrons, typically below about $40 \mathrm{eV}$. The phase retrieval methods available today for an IAP all rely on the SFA. In the FROG-CRAB method, additional approximations are imposed. To fully utilize the existing FROG phase retrieval algorithm, for example, the so-called central momentum approximation is used (see below). This approximation would limit the FROG-CRAB to attosecond pulses that have narrow spectral width, for pulses that have duration close to about 100 as and above. For broadband pulses, the central momentum approximation is no longer applicable. Three methods have been proposed beyond the FROG-CRAB: PROOF [11], PROBP [12], and VTGPA [13]. All of these algorithms are based on iterative methods. To "show" that the retrieved results are converged, the retrieved attosecond pulse is used to generate theoretical streaking spectra using the SFA. By comparing to the experimental electron spectra visually, it is often "concluded" that the retrieved results are correct and the pulse durations are reported.

In reality, it is very difficult to ascertain how accurately the spectral phase of an attosecond pulse has been retrieved. This difficulty increases as the spectral band extends beyond $100 \mathrm{eV}$. In our test with "experimental" data generated from the SFA model with known input pulses, it would often take tens of thousands of iterations to reach "convergence" for a known input pulse. The rate of convergence becomes much slower when the input pulse has larger chirp. We have found that for such broadband pulses, it is nearly impossible to distinguish the streaking spectra even when the spectral phases are significantly changed. In this paper, we show that 
the autocorrelation $(\mathrm{AC})$ of the photoelectron spectra reveals more clear variations. From the AC, we also define two metrics, called $V$ and $A$. These two metrics can be used to quantify the quality of the retrieved pulse. In addition, we found that the AC pattern can be used directly for the phase retrieval. Applying the PROBP method to the AC pattern, the revised retrieval method is found to converge to the correct answer much faster, and thus an accurate phase can be retrieved in a shorter time. In this paper, we illustrate how this method works for pulses that have bandwidth of about $30 \mathrm{eV}$ or less. Illustrations for water-window attosecond pulses with bandwidth above $100 \mathrm{eV}$ will be addressed in a future paper.

In the following, we first describe how the autocorrelation of the streaking spectra is calculated. We then examine how the shape of the AC pattern evolves as the spectral phase is varied. The AC patterns are analyzed through two metrics $V$ and $A$ quantitatively. We next use the PROBP [12,14,15] method to retrieve the spectral phase using the AC pattern instead of the streaking trace. This method is to be called PROBP-AC. We will show that PROBP-AC converges much faster than other existing methods that are based on retrieving from the streaking spectra. The two metrics $V$ and $A$ of the $\mathrm{AC}$ can also be used to evaluate the quality of agreement between the retrieved pulse and the "experimental" pulse. The AC pattern and the comparison of the PROBP-AC, PROBP, and FROG-CRAB methods are illustrated in examples. Furthermore, using the $V$ and $A$ metrics, we conclude that the intensity of the IR should not be too low (say, less than $10^{12} \mathrm{~W} / \mathrm{cm}^{2}$ ). We also show that the AC pattern is insensitive to the transition dipole such that the PROBP-AC method can also be used to retrieve attosecond pulses that generate low-energy photoelectron streaking spectra.

\section{THEORETICAL BACKGROUND AND THE RETRIEVAL METHOD}

\section{A. Strong-field approximation and the autocorrelation pattern}

The photoelectron spectrogram can be modeled by the SFA $[10,16]$ :

$$
\begin{aligned}
S(E, \tau)= & \mid \int_{-\infty}^{\infty} E_{\mathrm{XUV}}(t-\tau) d(p+A(t)) \\
& \times\left. e^{-i \varphi(p, t)} e^{i\left(\frac{p^{2}}{2}+I_{P}\right) t} d t\right|^{2},
\end{aligned}
$$

where $E=p^{2} / 2$ is the photoelectron energy and $\tau$ is the time delay between the two pulses. $E_{\mathrm{XUV}}(t)$ is the electric field of the XUV or the SXR pulse that is to be characterized. For simplicity we use XUV to include SXR, and IR to include mid-IR in the following unless otherwise specified. In Eq. (1), $d(p)$ is the transition dipole between the initial bound state and the final continuum state of the photoelectron, $I_{p}$ is the ionization potential of the target atom, and $\varphi(p, t)$ is the phase function. The vector potential of the IR is $A(t)=-\int_{-\infty}^{t} E_{\mathrm{IR}}\left(t^{\prime}\right) d t^{\prime}$. In Eq. (1) we assume that the polarization direction of the XUV, the IR, and the direction of the emitted electron are the same. Atomic units are used in this paper unless otherwise noted.

In the energy domain, an XUV pulse is expressed as

$$
E_{\mathrm{XUV}}(\Omega)=U(\Omega) e^{-i \Phi(\Omega)} .
$$

A single-color, multicycle IR field in the time domain is expressed as

$$
E_{\mathrm{IR}}(t)=f(t) \cos \left[\omega_{L} t+\varphi_{\mathrm{IR}}(t)\right] .
$$

In this work, we assume that the amplitude and phase of the transition dipole $d(p)$ are known, $d(p)$ is calculated from the single-active-electron model potential [17], and only the ionization from the outermost subshell is considered. The IR field has a Gaussian envelope and a zero carrier envelope phase. We also assume that the spectral amplitude of the XUV, i.e., $U(\Omega)$, is known since it can be extracted from XUV photoionization experiment without the IR.

From the spectrogram an autocorrelation is defined by the following:

$$
Q\left(\tau_{1}, \tau_{2}\right)=\int S\left(E, \tau_{1}\right) S\left(E, \tau_{2}\right) d E .
$$

The integration limits typically cover the whole photoelectron spectrum.

\section{B. Pulse retrieval based on the autocorrelation pattern}

In the simulation, the "experimental" spectrogram is obtained from Eq. (1) with known input XUV and IR fields. For simplicity in the retrieval we assume the IR field is known in this work. Therefore our goal is limited to retrieve the phase of the XUV, i.e., $\Phi(\Omega)$. This unknown function is expanded in terms of $B$-spline basis functions.

In general, a smooth function $f(x)$ can be expanded as

$$
f(x)=\sum_{i=1}^{n} g_{i} B_{i}^{k}(x),
$$

where $g_{i}$ are expansion coefficients; $i$ is the index of the $B$-spline function. The $k$ th-order $B$-spline functions $B_{i}^{k}(x)$ are defined through

$$
\begin{gathered}
B_{i}^{1}(x)= \begin{cases}1, & x_{i} \leqslant x \leqslant x_{i+1}, \\
0, & \text { otherwise },\end{cases} \\
B_{i}^{k}(x)=\frac{x-x_{i}}{x_{i+k-1}-x_{i}} B_{i}^{k-1}(x)+\frac{x_{i+k}-x}{x_{i+k}-x_{i+1}} B_{i+1}^{k-1}(x) .
\end{gathered}
$$

Here $\left\{x_{i}\right\}$ are the knot points. If there are $n B$-spline basis functions of the order $k$, the total number of knot points is $n+$ $k$. The $B$-spline function is a powerful tool for fitting a smooth function [18], and it has been widely used in computational physics.

In our situation, we define the guessed $B$-spline expansion coefficients for the spectral phase as $\left\{b_{i}\right\}$. From these coefficients the guessed spectral phase of the XUV can be constructed, and then we calculate the spectrogram by Eq. (1) and the AC pattern by Eq. (4). The expansion coefficients $\left\{b_{i}\right\}$ are to be determined when the error function defined by

$$
E\left[b_{i}\right]=\sum_{k, l}\left[Q_{0}\left(\tau_{k}, \tau_{l}\right)-Q_{1}\left(\tau_{k}, \tau_{l}\right)\right]^{2}
$$

reaches the minimum, where $Q_{0}$ and $Q_{1}$ are the input and reconstructed AC patterns, respectively. In the numerical computation we discretize the spectrogram $S(E, \tau)$ and the AC pattern $Q\left(\tau_{1}, \tau_{2}\right)$ on grid points. Typically we chose 100 to 500 points in time delay and 100 points in energy. We use 

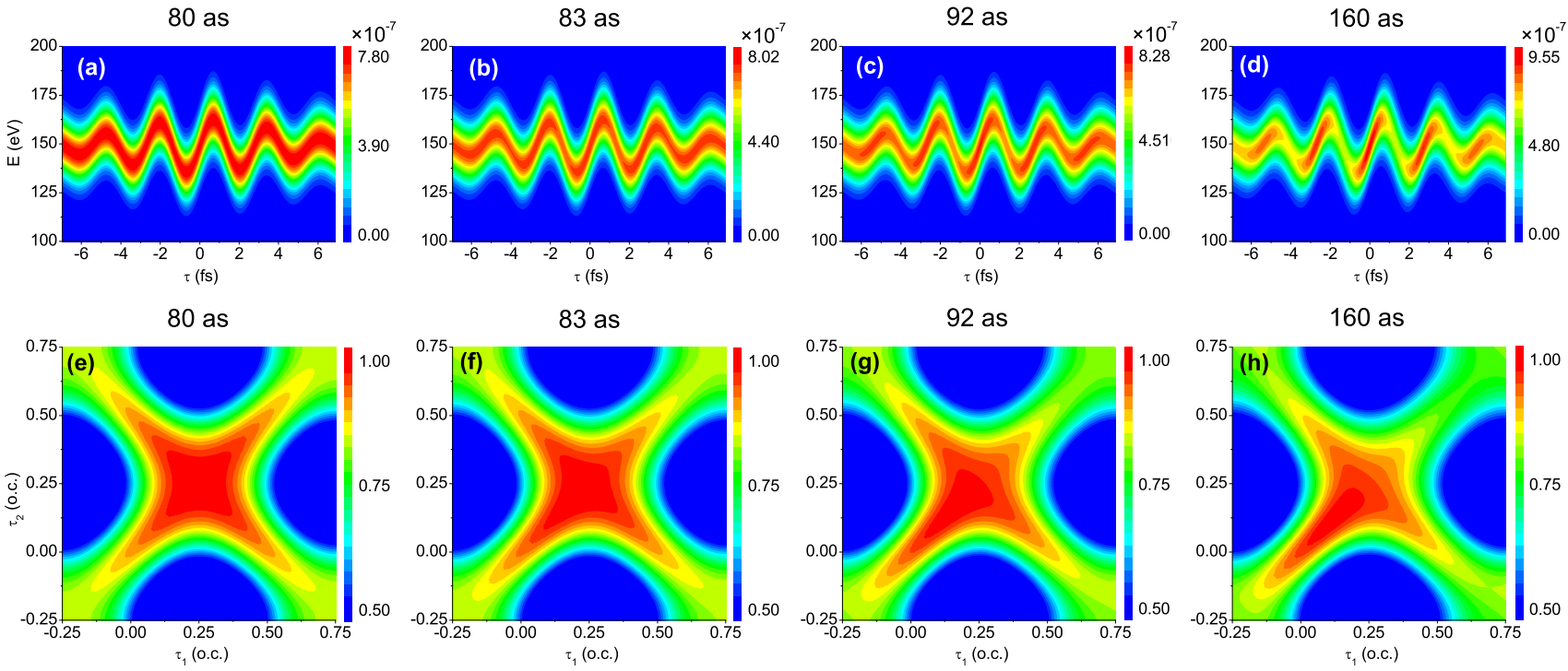

FIG. 1. SFA-simulated spectrogram (upper panel) and autocorrelation pattern (lower panel) for the TL and three linearly chirped XUV pulses. The four XUV pulses are centered at $164 \mathrm{eV}$ with a bandwidth of $23 \mathrm{eV}$. The FWHM durations of these pulses are (a) 80 , (b) 83 , (c) 92, and (d) 160 as, respectively. The autocorrelation patterns (e)-(h) are plotted within one optic cycle of the IR field.

the genetic algorithm (GA) to find the optimal parameters $\left\{b_{i}\right\}$ that would minimize Eq. (8). The GA runs a large number of generations (typically 300 to 500 generations) until convergence is achieved. The optimal parameters $\left\{b_{i}\right\}$ are then used to reconstruct the XUV pulse.

We describe how to choose the GA and $B$-spline parameters in more detail in the Appendix.

\section{AUTOCORRELATION PATTERNS OF DIFFERENT XUV PULSES WITH ATTOCHIRP}

\section{A. Streaking spectra and autocorrelation pattern}

Consider an XUV pulse with a Gaussian spectral amplitude $U(\Omega)=U_{0} e^{-2 \ln 2 \frac{\left(\Omega-\Omega_{0}\right)^{2}}{\left(\Delta_{\Omega}\right)^{2}}}$ and a quadratic spectral phase
$\Phi(\Omega)=a_{2} \frac{\left(\Omega-\Omega_{0}\right)^{2}}{(\Delta \Omega / 2)^{2}}$. Here we use $\Omega_{0}=164 \mathrm{eV}$ as the central photon energy and $\Delta \Omega=23 \mathrm{eV}$ as the FWHM (full width at half maximum) bandwidth, which can support a transformlimited (TL) pulse (corresponding to $a_{2}=0$ ) of 80 as in the FWHM duration. The coefficient $a_{2}$ is a measure of the attochirp, or equivalently the duration of the chirped pulse compared with the TL duration. Figures 1(a)-1(h) show the spectrograms and correlation patterns for the TL pulse and three chirped pulses with different durations. The spectrograms are simulated using SFA according to Eq. (1). The IR used here is $800 \mathrm{~nm}$ in wavelength, $5.7 \mathrm{fs}$ in FWHM duration, and $2.5 \times 10^{12} \mathrm{~W} / \mathrm{cm}^{2}$ in peak intensity. The autocorrelation patterns are calculated based on Eq. (4) with $\tau_{1}, \tau_{2}$ within one optical cycle of the IR field. In this example, while one can
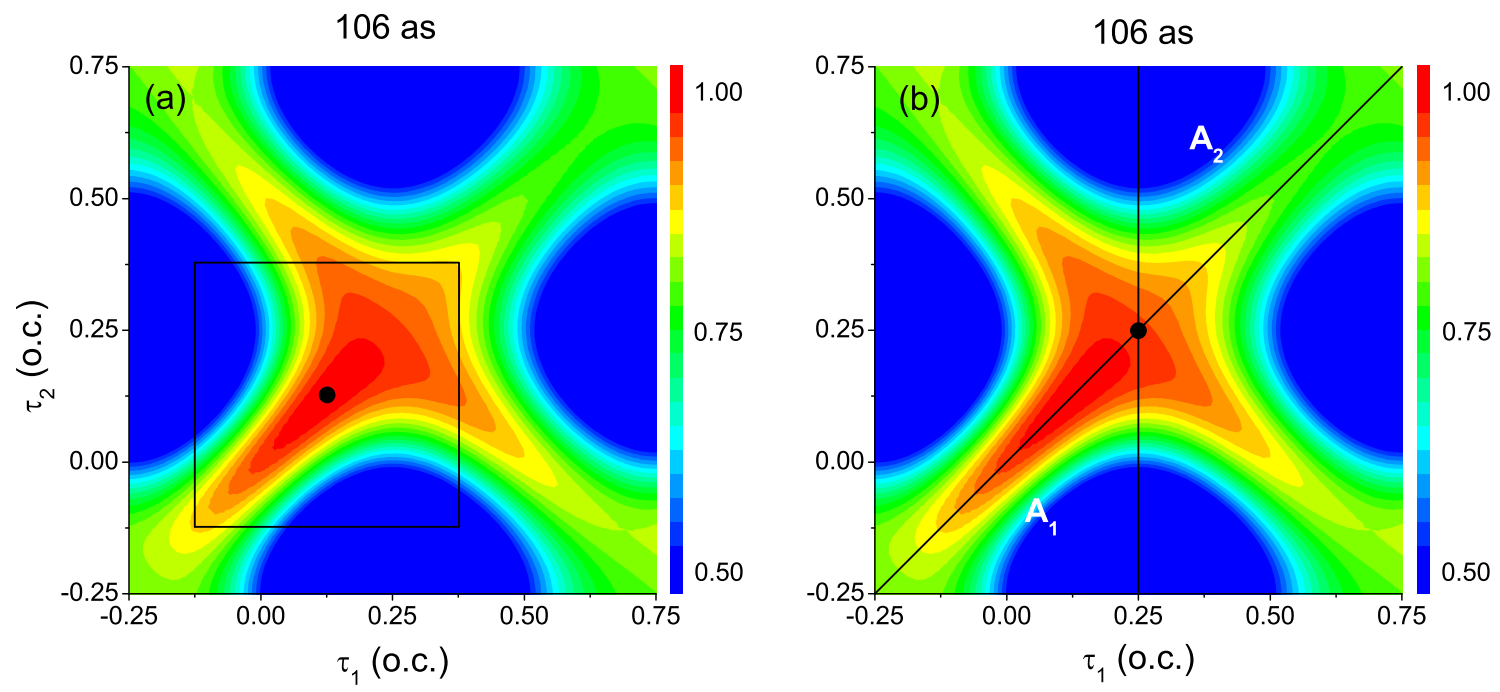

FIG. 2. Autocorrelation pattern over one optical cycle and the definition of (a) the volume $(V)$ and (b) asymmetry $(A)$ of the autocorrelation over half an optical cycle. The black dot in Fig. 2(a) is the maximum point of $Q\left(\tau_{1}, \tau_{2}\right)$ for a 106-as chirped pulse. In Fig. 2(b), the center of $\mathrm{AC}$ is at $(0.25,0.25)$ which is the position of the maximum defined with respect to TL pulse. 


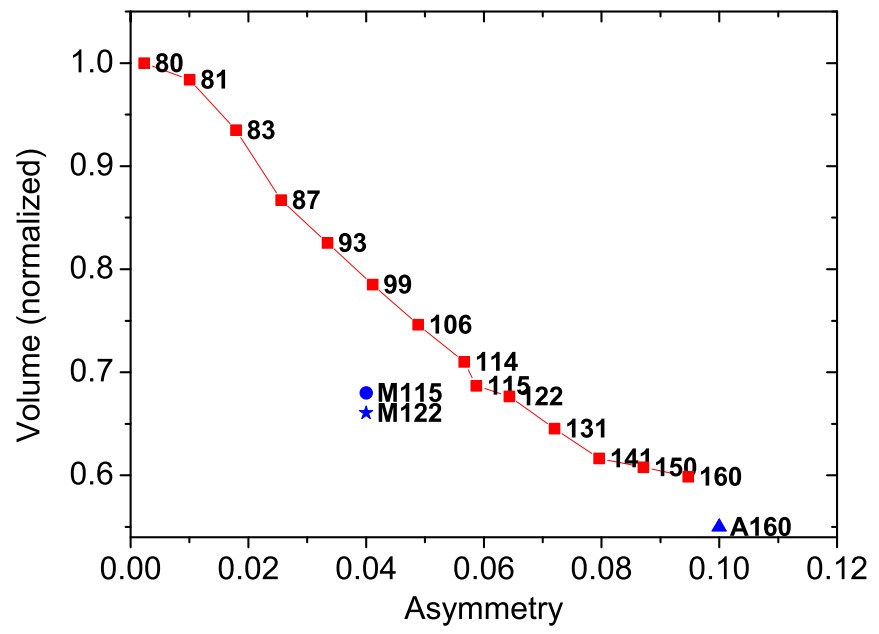

FIG. 3. The two metrics of the autocorrelation pattern. For each streaking trace, its $V$ and $A$ values are given by a point on the $V$-A plane. These points form a smooth curve if the pulses are linearly chirped. For pulses that are nonlinearly chirped, they would lie below this curve. Three such pulses M115, M122, and A160 are shown.

see that the streaking spectra display some fine differences (the yield decreases in the half cycle when the electron energy drops with increase of time delay) as the duration of the XUV pulse is increased, the change is much more pronounced in the AC pattern (the strength of the northeast quarter of each pattern drops rapidly). Also, from the AC pattern $Q\left(\tau_{1}, \tau_{2}\right)$ one can clearly distinguish the chirped pulse from the TL pulse. The TL pulse yields a squarelike pattern with high symmetry, while the chirped pulse gives an asymmetric pattern. Therefore if one wants to confirm that the XUV pulse is nearly transform-limited, one only needs to calculate its AC pattern to show that it is nearly symmetric.

\section{B. The $V$ and $A$ metrics of the AC pattern}

In order to further distinguish different chirps from the AC pattern, we introduce two quantitative metrics. The metric "volume" $(V)$ is defined as $V=\iint Q\left(\tau_{1}, \tau_{2}\right) d \tau_{1} d \tau_{2}$ and the normalized $V$ is given by

$$
V_{\text {normalized }}=\frac{\iint Q\left(\tau_{1}, \tau_{2}\right) d \tau_{1} d \tau_{2}}{\iint Q^{T L}\left(\tau_{1}, \tau_{2}\right) d \tau_{1} d \tau_{2}},
$$

where the integral is over a square box centered at the peak of $Q\left(\tau_{1}, \tau_{2}\right)$ with the side length equal to one-half optical cycle of the IR. For this TL case, the $Q^{(T L)}\left(\tau_{1}, \tau_{2}\right)$ peak point is at $(0.25,0.25)$, as indicated in Fig. 2(a). For the chirped pulse, we calculate the asymmetry $A$ of the $\mathrm{AC}$ with respect to this center $(0.25,0.25)$ of the peak of the TL pulse; see Fig. 2(b).
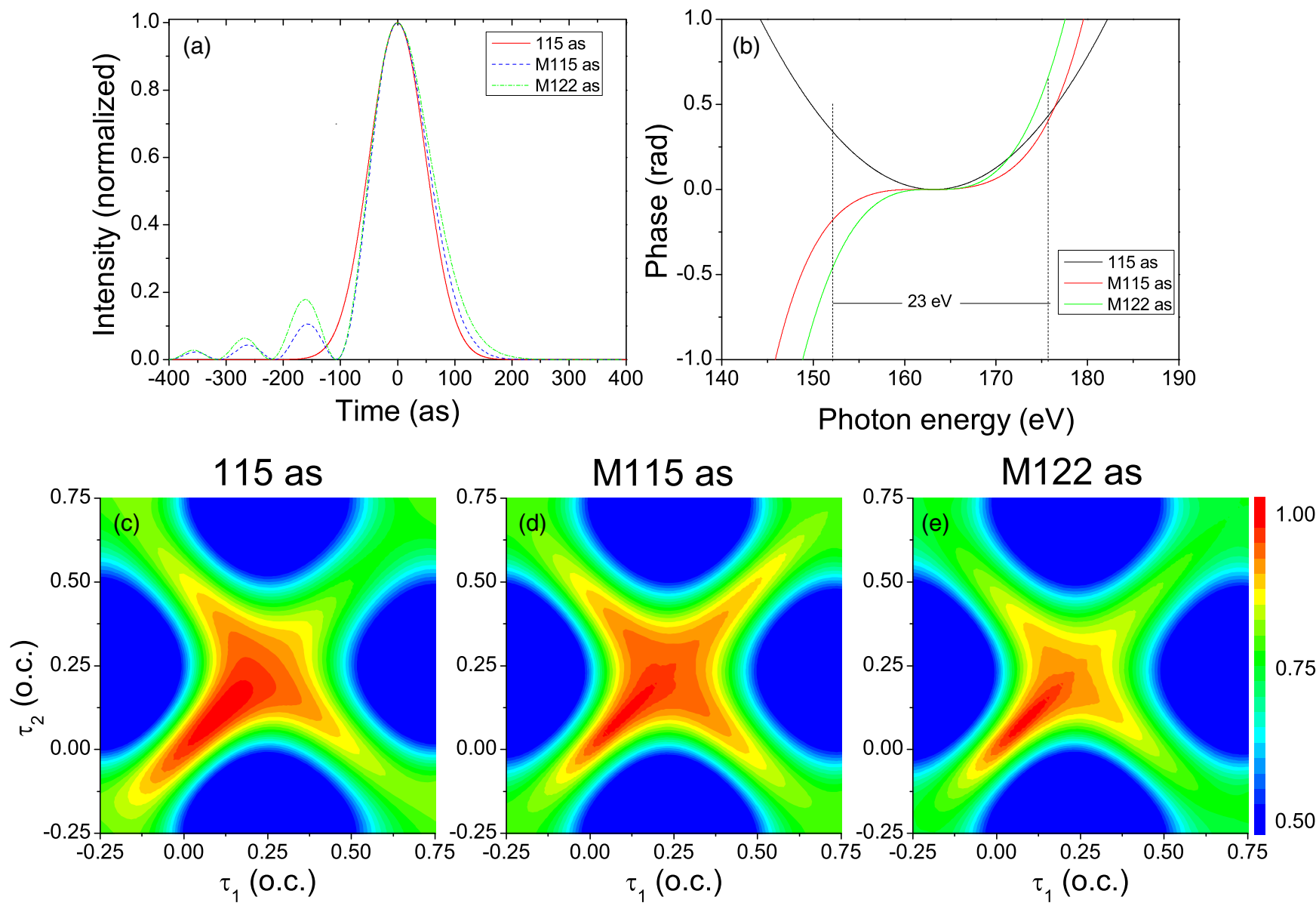

FIG. 4. Temporal envelope of intensity (a) and spectral phase (b) for the three pulses, 115, M115, and M122 (upper panel). Autocorrelation patterns (c)-(e) for the three pulses (lower panel) are shown. 

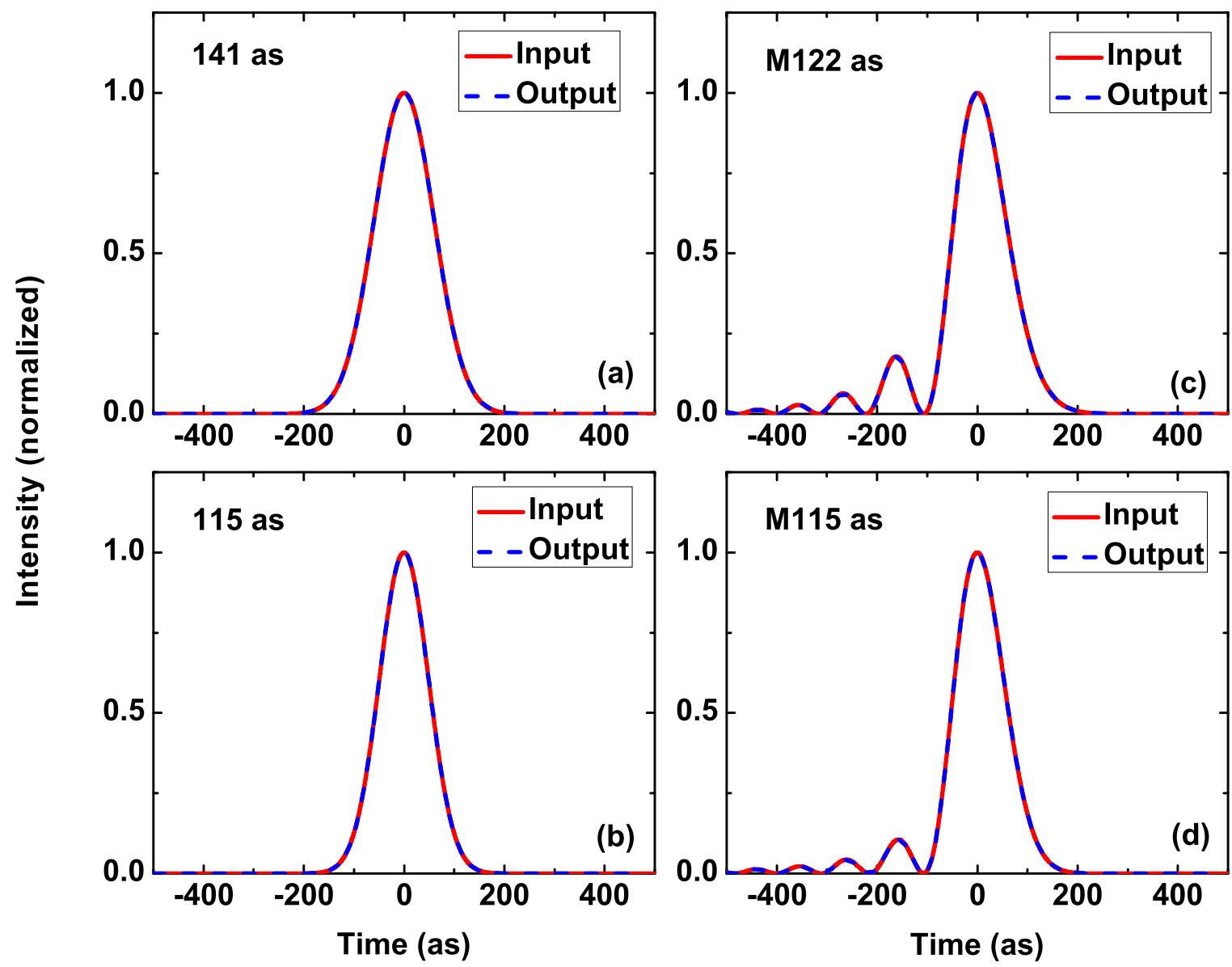

FIG. 5. Comparison between the input and the retrieved XUV intensity, for the case of linearly chirped XUV pulses with durations of (a) 141 and (b) 115 as, and the nonlinear chirped pulses (c) M122 and (d) M115. The retrieval is based on fitting the autocorrelation pattern. The output is from 500 iterations.

Here $A$ is given by

$$
A=\frac{Q_{A_{1}}-Q_{A_{2}}}{Q_{A_{1}}+Q_{A_{2}}},
$$

where $Q_{A_{1}}$ and $Q_{A_{2}}$ are the integrals of $Q\left(\tau_{1}, \tau_{2}\right)$ over the triangle region $A_{1}$ and $A_{2}$, respectively, as indicated in Fig. 2(b). By changing the chirp parameters of the XUV pulse one can calculate the corresponding $\mathrm{AC}$ pattern and the two metrics. Figure 3 shows the metric volume $V$ (vertical axis) and asymmetry $A$ (horizontal axis) for several chirped pulses whose FWHM durations are given as numbers near the data markers. For linearly chirped pulses (red squares) one can see that as the pulse duration increases the volume decreases and the asymmetry increases monotonically. If one knows that the pulse is linearly chirped, then from the $(V, A)$ values of the experimental AC pattern, one can read off the pulse duration from the red curve in Fig. 3.

A few words are needed to describe how we construct the AC pattern. It is noted that we set the time delay to be zero when the peaks of the XUV and the IR pulses coincide in time. As the spectral phase of the XUV pulse is changed, its peak position in time is also changed. Figure 2(a) shows that the time shift of the peak of a 106-as pulse is at 0.125 optical cycle compared to the peak of the 80 -as TL pulse. In comparing the streaking spectra, the absolute signal in the experimental streaking spectra is normalized to 1.0. The same normalization is also used for each streaking spectrogram calculated from the SFA.

\section{AC patterns for nonlinearly chirped pulses}

In general, an XUV pulse has a more complicated spectral phase. First, we consider that the spectral phase of an XUV pulse from high-order harmonic generation in a gas medium is well behaved such that it can be expanded by $\Phi(\Omega)=a_{2} \frac{\left(\Omega-\Omega_{0}\right)^{2}}{(\Delta \Omega / 2)^{2}}+a_{3} \frac{\left(\Omega-\Omega_{0}\right)^{3}}{(\Delta \Omega / 2)^{3}}+a_{4} \frac{\left(\Omega-\Omega_{0}\right)^{4}}{(\Delta \Omega / 2)^{4}}+a_{5} \frac{\left(\Omega-\Omega_{0}\right)^{5}}{(\Delta \Omega / 2)^{5}}$, where we set the phase to be zero at the center of the spectral amplitude of the pulse. For such general cases, the $V$ and $A$ values of the $\mathrm{AC}$ pattern will not fall on the smooth curve in Fig. 3. In Fig. 4, we construct two pulses with nonlinear chirps, called M115 and M122, respectively, to be compared to the one called 115 which has linear chirp only. All three pulses share the same spectral amplitudes. The spectral phases of the three pulses are shown in Fig. 4(b), where each has FWHM spectral width of $23 \mathrm{eV}$. The temporal profiles of the three pulses are shown in Fig. 4(a). Note that the FWHM of the main peaks are very close to each other to within $2 \%$, but the FWHM of the main peak alone does not correctly represent M115 and M122 pulses since both pulses have non-negligible subpeaks beyond the main peak. In fact, for 
TL 160 as

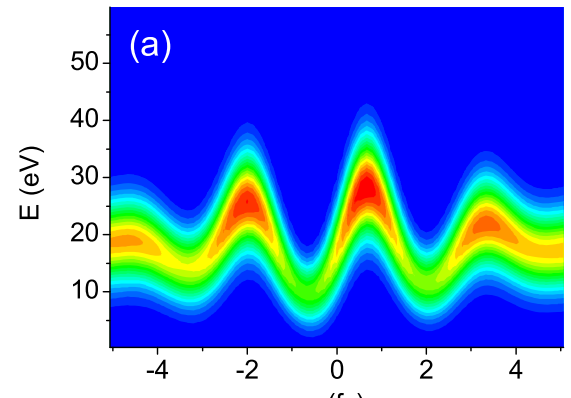

$\tau(\mathrm{fs})$

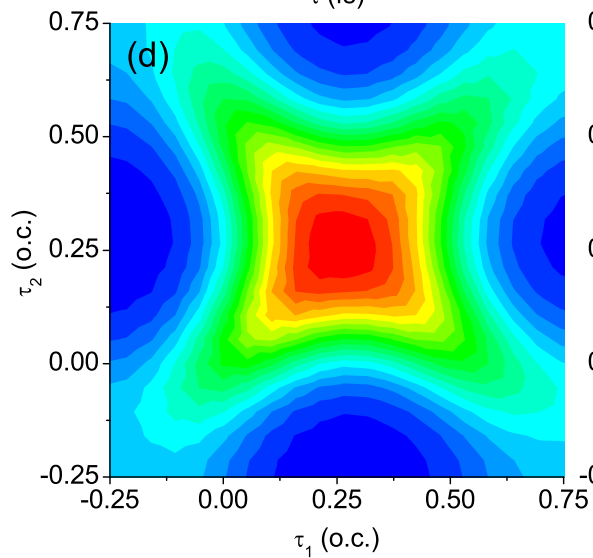

200 as

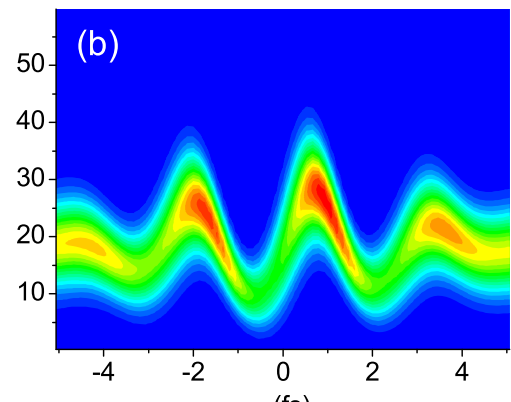

$\tau(\mathrm{fs})$

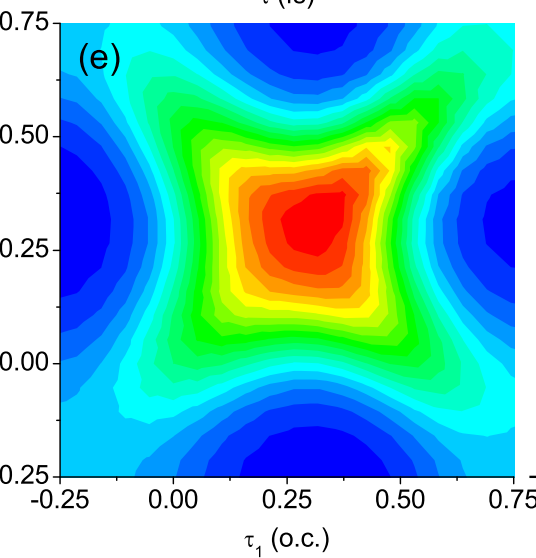

250 as

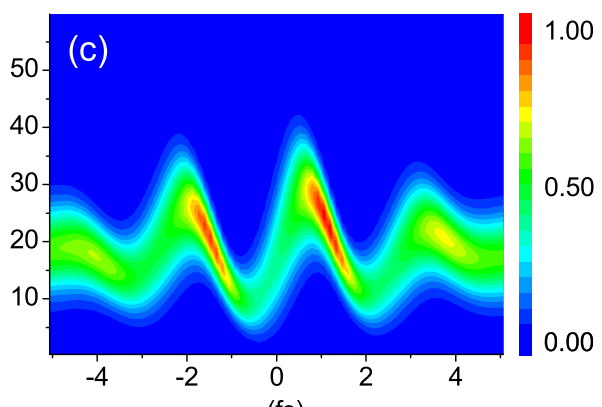

$\tau(\mathrm{fs})$

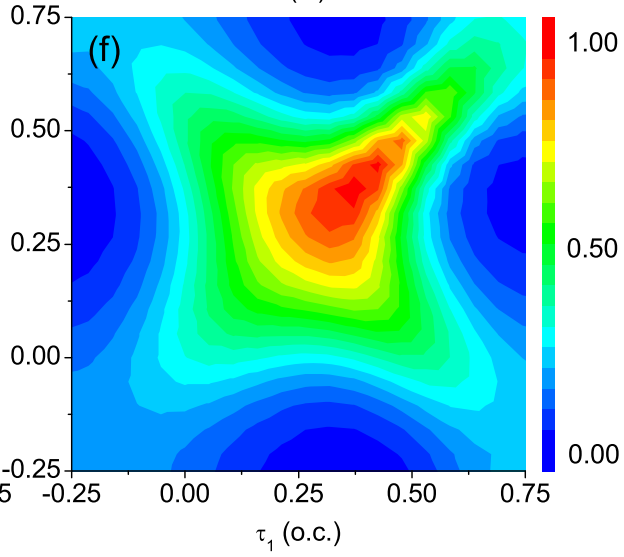

FIG. 6. TDSE-simulated Ne spectrograms [(a)-(c)] and the corresponding AC patterns [(d)-(f)]. The three XUV pulses are centered at 40-eV photon energy, with an FWHM bandwidth of $11.5 \mathrm{eV}$. The TL pulse has a duration of 160 as, while the two linearly chirped pulses are 200 as and 250 as, respectively.
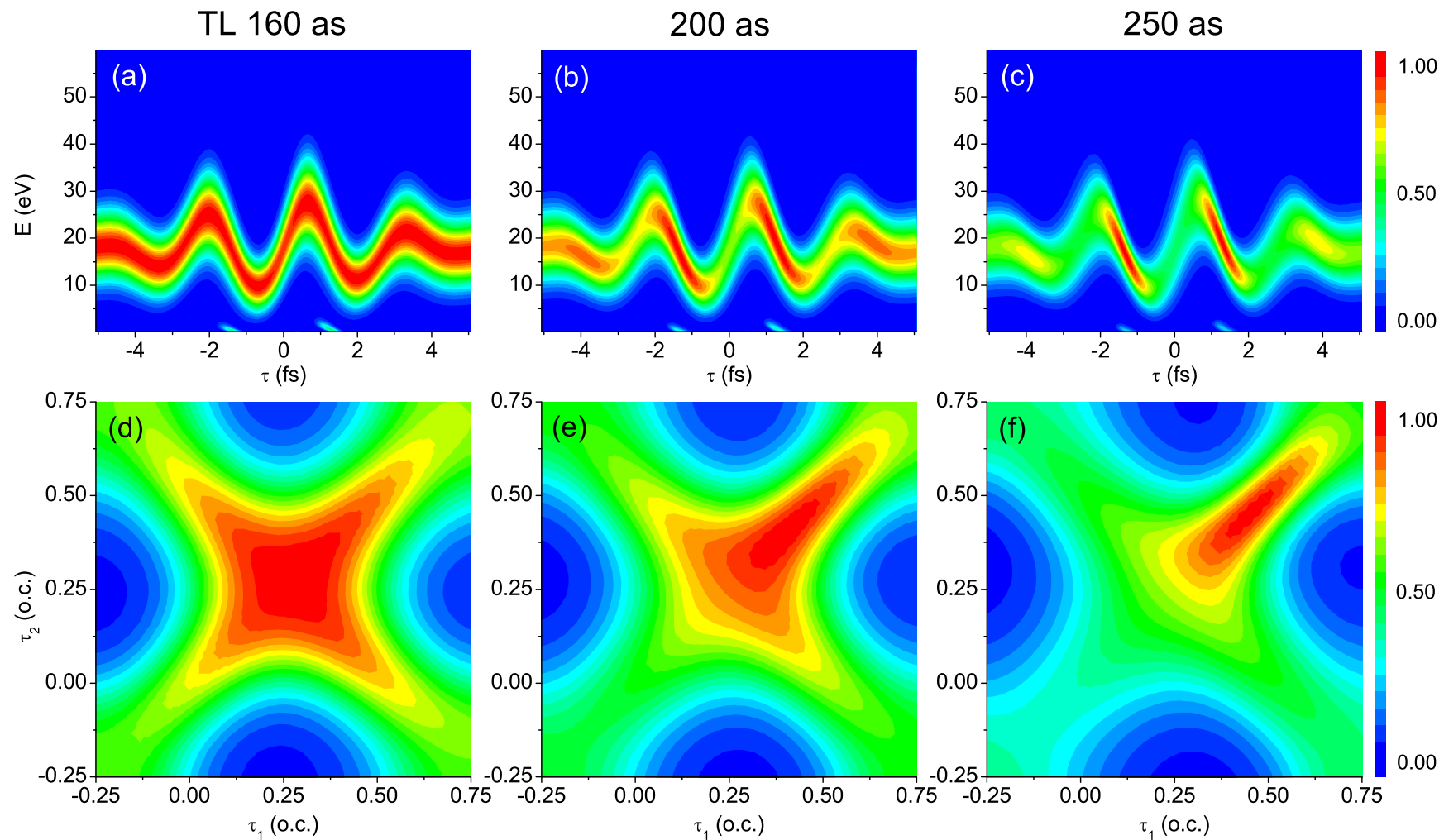

FIG. 7. SFA-simulated Ne spectrograms [(a)-(c)] and the corresponding AC patterns [(d)-(f)]. Parameters are the same as in Fig. 6. 

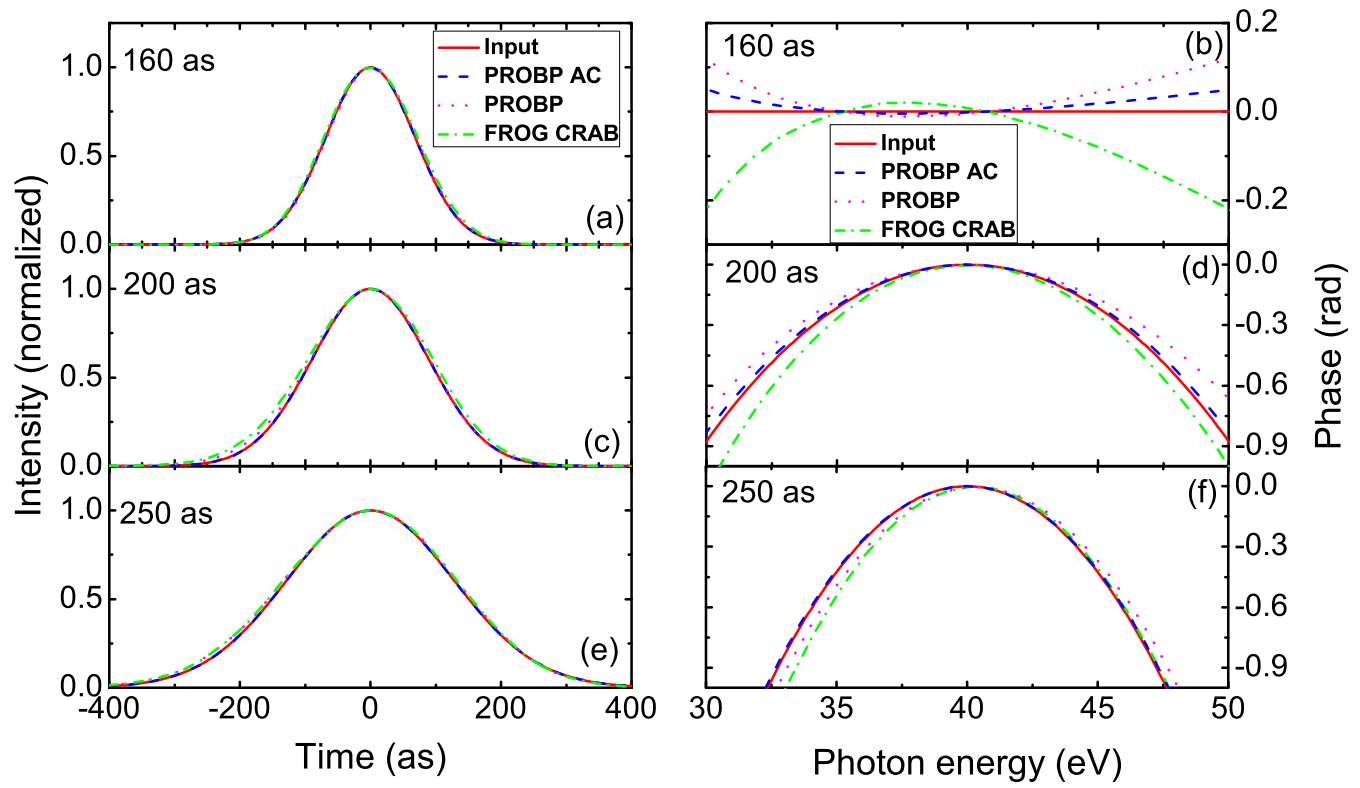

FIG. 8. Retrieval results for the (a)-(b) 160-as, (c)-(d) 200-as, and (e)-(f) 250-as XUV pulses from the TDSE-simulated data in Fig. 6. Three retrieval methods are shown here. The results show the increasing accuracy from the FROG-CRAB, PROBP, and PROBP-AC methods, as compared to the input pulse.

M122, the electric field magnitude at the second peak is $40 \%$ of the field at the main peak. From the constructed AC patterns, the three different pulses can be easily distinguished; see Figs. 4(c), 4(d) and 4(e). Even the $(V, A)$ values can be used to distinguish the three pulses; see the labels in Fig. 3 for M115 and M122. Both of them are located away from the red curve where the 115 pulse is located. Thus we know both M115 and M122 have nonlinear chirps, but the main peak has FWHM of 115 as and 122 as, respectively. For pulses with nonlinear chirps, multiple parameters are needed to characterize the spectral phase within the spectral width of the pulse; thus accurate retrieval of the spectral phase in general will take more effort, and a single parameter like pulse duration is unable to characterize the pulse.

\section{PROBP-AC algorithm for attosecond pulse characterization}

In Ref. [12] we used the PROBP (phase retrieval of broadband pulses) method to obtain the spectral phase of a broadband pulse using the streaking trace directly. This method is an improvement over the FROG-CRAB since it does not employ the central momentum approximation. Here we use the AC pattern of the streaking spectra and the same optimization method of PROBP to extract the spectral phase; see Eq. (8). This method is called PROBP-AC. Figures 5(a)-5(d) show that the input pulses can be accurately retrieved using the PROBP-AC easily. For the pulses shown here, it takes about 500 iterations to get converged results, much faster than the PROBP method of several thousand iterations.

\section{AUTOCORRELATION PATTERNS FROM TDSE SIMULATION COMPARED TO SFA}

So far we use SFA to simulate the experimental spectrogram. It is well known that the SFA is not accurate in the low-energy region. In this section, we use the streaking spectra generated by solving the time-dependent Schrödinger equation (TDSE) and take the results as the experimental data. The resulting streaking spectra and the AC patterns are shown in Figs. 6(a)-6(f). The spectral amplitude takes a Gaussian form with $\Omega_{0}=40 \mathrm{eV}$ and $\Delta \Omega=11.5 \mathrm{eV}$ which can support a TL pulse with the duration of 160 as. We include three XUV pulses, a TL pulse, and two linearly chirped pulses, with the durations of 160 as, 200 as, and 250 as, respectively. Ne is chosen as the target gas. The IR field is $800 \mathrm{~nm}$ in wavelength, $4.5 \mathrm{fs}$ in FWHM duration, and $1.0 \times 10^{13} \mathrm{~W} / \mathrm{cm}^{2}$ in peak intensity. The same parameters are also used to calculate the streaking spectra using the SFA equation and the AC patterns. The SFA results are shown in Figs. 7(a)-7(f).

Comparing the TDSE with the SFA results, it is quite clear that the streaking spectra do not agree very well. Can the TDSE streaking spectra or their AC patterns be used to extract accurate XUV pulses, knowing that all the retrieval methods rely on Eq. (1) which gives the spectra shown in Fig. 7? The retrieved results are shown in Fig. 8 where the left panels compare the XUV intensities in the time domain and the right panels compare the spectral phases. In Figs. 8(a), 8(c) and 8(e), all three input pulses in the time domain are accurately retrieved by the three methods - the FROG-CRAB, the PROBP, and the PROBP-AC. For Figs. 8(b), 8(d) and

TABLE I. Comparison of the number of iterations and computing times used for the three different retrieval methods.

\begin{tabular}{lrc}
\hline \hline & Iterations & Calculation time \\
\hline PROBP-AC & 500 & 20 minutes \\
PROBP & 5000 & 2 hours \\
FROG-CRAB & 100000 & 1 day \\
\hline
\end{tabular}


8(f), the retrieved spectral phases in the energy domain are compared. Near the center of the spectral domain (where we set the phase to zero at the center of the spectral amplitude), all the phases from the three methods agree with the input value, but upon closer look, the PROBP-AC is the most accurate one, while the FROG-CRAB is the worst. In addition, in terms of rate of convergence or computer time, the PROBP-AC method is far superior. For typical pulses like those shown in Fig. 8, the number of iterations and the computer times are listed in Table I. Clearly the PROBP-AC method proposed here is much faster.

Based on the extracted temporal profiles of the three pulses shown in Fig. 8, one may say that the XUV pulses can be accurately retrieved even for low-energy photoelectrons where the streaking spectra calculated using SFA are known to be inaccurate. However, the accuracy here is relative. From the right panel of Fig. 8 we can extract the error of the phase difference between $40 \mathrm{eV}$ (where the phase is set to zero) and $35 \mathrm{eV}$. Based on the numerical values calculated, we found that the error of the phase difference between 35 and $40 \mathrm{eV}$ (FWHM is $11 \mathrm{eV}$ ) is about 0.1 radians for FROG-CRAB and 0.01 radians for the PROBP-AC. For an error of 0.1 radians between the two energy points, it amounts to an error of about 12 as in time delay (or group delay in optics). This sets the stringent condition needed for retrieving very accurate spectral phase from the experimental data. Based on its fast convergence and more accurate phase retrieval, the PROBP-AC method should be used to replace the FROG-CRAB method even for the narrowband pulses, as the present example demonstrated. The well-cited photoionization time-delay difference from $2 s$ and $2 p$ levels in Ne reported in Schultze et al. [19] and Wei et al. [20] probably should be reexamined again with our retrieval method.

We have also applied the PROBP-AC method to retrieve an attosecond pulse where the XUV phase is not given by a polynomial expansion. In Fig. 9, we provide such an example. The AC pattern for a chirped pulse with arbitrary phase is presented in Fig. 9(a). The parameters of $V$ and $A$ for this pulse (called A160) are 0.55 and 0.1 , respectively. On the $V$ - $A$ plane, this A160 pulse is located outside the curve for the linearly chirped pulse; see Fig. 3. The input XUV phase and temporal envelope are shown in Figs. 9(b) and 9(c). The figures also show that they are accurately retrieved by the PROBP-AC method.

We use this example to illustrate the computational details of our retrieval method in the Appendix. In this case, the GA parameters we use are provided as follows: $x_{\min }=0.0$, $x_{\max }=100.0, L=15, P_{\text {cross }}=0.5, n=7$, and $N=20$. For $B$-spline functions, we use $k=7$ and $n+k=14$. After about 500 generations, we get converged results.

\section{EFFECT OF IR WAVELENGTH AND INTENSITY, AND DIPOLE OF THE ATOM}

The sensitivity of the AC pattern to attochirp depends on the IR pulse parameters, e.g., wavelength and intensity. Consider a linearly chirped XUV pulse, with $\Omega_{0}=164 \mathrm{eV}$ and $\Delta \Omega=23 \mathrm{eV}$. We can define a parameter $\gamma$ which is the ratio of the duration of the chirped pulse to the duration of the TL pulse ( 80 as in this case) to indicate the amount
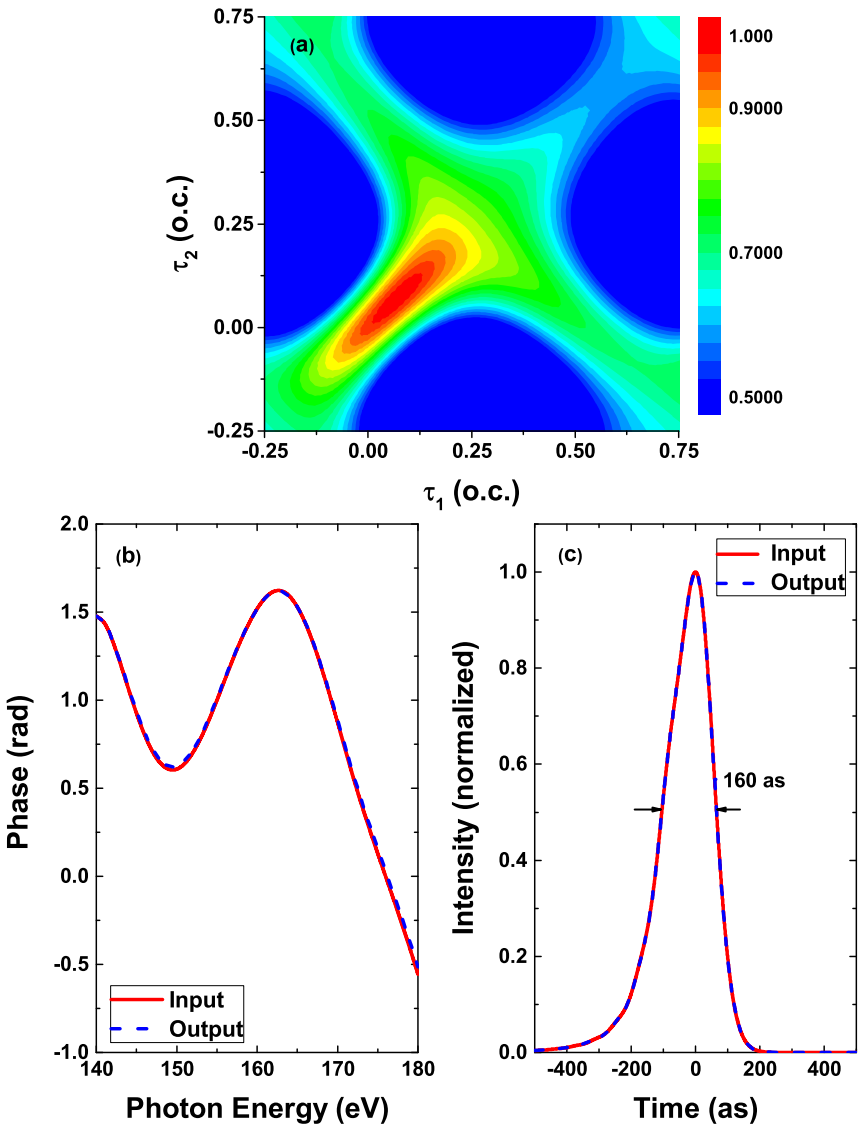

FIG. 9. AC pattern (a) for a 160-as chirped pulse with arbitrary phase is shown. Comparison between the input and the retrieved XUV phase (b) and intensity (c). The laser parameters are the same as that in Fig. 5 except that a more complex phase is used as input.

of attochirp. In Fig. 10(a) we plot the dependence of the metric volume from the AC pattern versus $\gamma$. On the left panel we fix the IR wavelength to $800 \mathrm{~nm}$ and change its intensity. It is clear that the slope decreases with decreasing IR intensity. This means the streaking contrast is weaker at lower IR intensity, thus making the retrieval method less sensitive to the spectral phase.

In Fig. 10(b) we fix the IR intensity to $2.5 \times 10^{12} \mathrm{~W} / \mathrm{cm}^{2}$ and change its wavelength. The metric volume decreases monotonically with $\gamma$, and the slope reveals the sensitivity to wavelength. Larger sensitivity can lead to faster convergence and better performance of the retrieval method. We can see the best sensitivity happens when using 800-nm and 1200-nm IR wavelengths, while the performance becomes lower when we either increase the wavelength to $1800 \mathrm{~nm}$ or decrease the wavelength to $400 \mathrm{~nm}$. The conclusion may differ somewhat if both wavelength and intensity of the IR are varied at the same time.

Finally we also check the sensitivity of the AC patterns and the PROBP-AC method to the magnitude of the atomic transition dipole. The dependence is very small. In Table II we show four examples and express the $(V, A)$ values obtained for the four different pulses, using the actually calculated atomic dipole as well as taking the dipole to be independent of energy. Table II shows that the $(V, A)$ values for these 

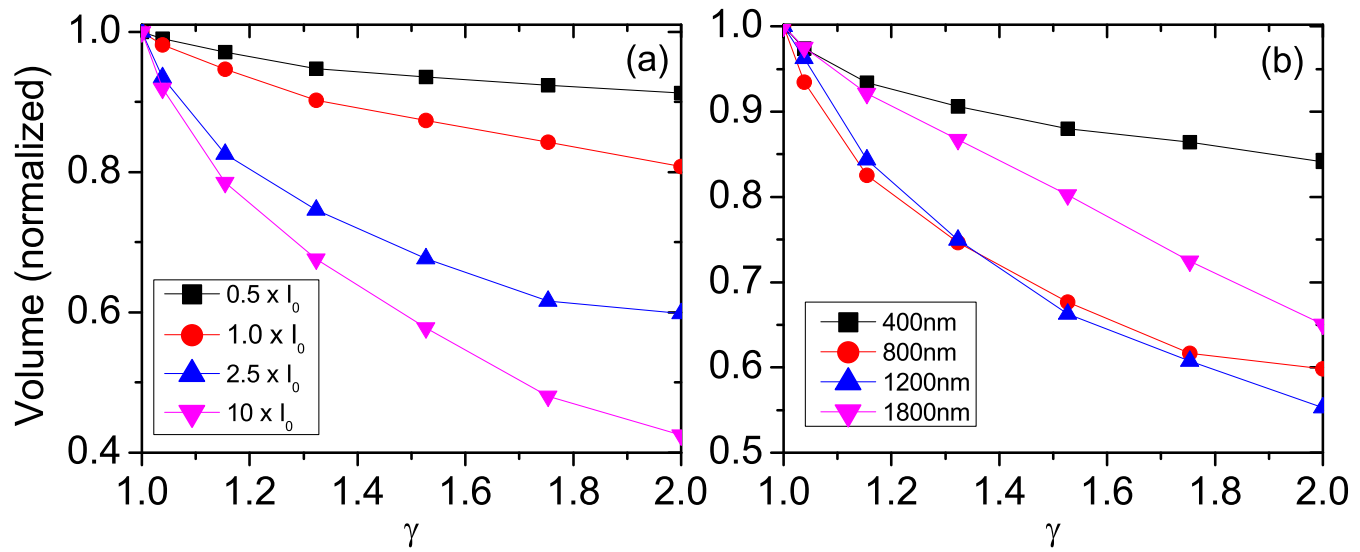

FIG. 10. (a) Dependence of the metric volume on the XUV chirp for various IR intensities. Only pulses with linear chirp are considered. The horizontal axis gives the ratio of the pulse duration in units of the duration of the TL pulse. The XUV pulses are centered at $164 \mathrm{eV}$ with a bandwidth of $23 \mathrm{eV}$. The TL pulse duration is 80 as. (b) The dependence of the metric volume vs wavelength of the IR at a fixed intensity of $2.5 \times I_{0}, I_{0}=10^{12} \mathrm{~W} / \mathrm{cm}^{2}$.

pulses are essentially the same. Thus accurate atomic dipole moment is not needed for the retrieval of the XUV pulses in streaking experiments, at least for the narrowband XUV pulses of spectral widths of tens of eVs.

\section{SUMMARY}

In this article, we reported a method of retrieving the spectral phase of an IAP in the XUV to the SXR region. We note that the spectral amplitude of an IAP can be readily obtained from the photoelectron spectrum by ionizing a rare gas atom using the XUV or the SXR pulse alone. To retrieve the spectral phase, several algorithms have been proposed so far, including FROG-CRAB, PROBP, and VTGPA, by analyzing the streaking spectrogram generated by photoionization of an atom by the XUV pulse in the presence of a time-delayed infrared pulse. Unlike all of these previous works where the spectral phase is retrieved iteratively from analyzing the streaking trace, in this article we retrieve the spectral phase from analyzing the autocorrelation $(\mathrm{AC})$ of the trace, and our method is called PROBP-AC. We found that this method converges faster and that it is capable of retrieving the correct spectral phase. By comparing the AC generated from the retrieved pulse with the AC from the "experimental" trace, we are able to visualize clearly how good the retrieved pulse is. In addition we defined two parameters $V$ and $A$ from the AC pattern. The agreement of these two numbers from

TABLE II. Comparison of $(V, A)$ values of the AC patterns of the streaking trace for four different XUV pulses, calculated using Eq. (1) where the transition dipole is from the actual $\mathrm{Kr}$ target atom or approximated by a constant.

\begin{tabular}{lcc}
\hline \hline & $(V, A)$ (actual dipole) & $(V, A)($ constant dipole) \\
\hline 106 as & $(0.75,0.049)$ & $(0.75,0.048)$ \\
115 as & $(0.68,0.058)$ & $(0.69,0.057)$ \\
141 as & $(0.62,0.079)$ & $(0.63,0.078)$ \\
M115 as & $(0.68,0.040)$ & $(0.73,0.030)$ \\
\hline \hline
\end{tabular}

the retrieved pulse and from the "experiment" also provides another check on how accurately the pulse is retrieved.

In this article, we present the PROBP-AC method and use examples from attosecond pulses that have spectral bandwidth of about $30 \mathrm{eV}$. We compare this method against the ones previously used like PROBP and FROG-CRAB to establish that our method is more efficient and more accurate. The PROBP-AC method is particularly more powerful than other prevailing retrieval methods for pulses that have bandwidth higher than $100 \mathrm{eV}$. This topic will be addressed in a separate publication.

\section{ACKNOWLEDGMENTS}

This research was supported in part by the Chemical Sciences, Geosciences, and Biosciences Division, Office of Basic Energy Sciences, Office of Science, US Department of Energy, under Grant No. DE-FG02-86ER13491. W.Y. would also like to acknowledge partial support by the Chinese Scholarship Council (CSC), and by the National Natural Science Foundation of China under Grant No. 11604131.

\section{APPENDIX: HOW TO CHOOSE GA AND B-SPLINE PARAMETERS}

The genetic algorithm (GA) is a heuristic search method used for solving a multidimensional optimization problem. The algorithm is introduced based on the concept of natural selection in Darwin's evolutionary theory. The general scheme of searching the optimal solution in the GA involves the following steps: initialization of population for the first generation, fitness evaluation of the individuals, selection of parents based on fitness, and crossover (sometimes called recombination) and mutation for producing offspring. A new generation is then produced. If the fitness of the best individual in the new generation does not meet the requirement of termination, we repeat the same procedure until the optimal solution is found.

The genetic algorithm has been widely applied in various problems. Here, we focus and elaborate on the application of the genetic algorithm in our phase retrieval problem, i.e., 
PROBP-AC. In PROBP-AC, we expand the unknown phase of the XUV pulse in a set of $B$-spline basis functions (with size $n$ and order $k$ ), as is written in Eq. (5). So the goal is to find the expansion coefficients $\left\{b_{i}\right\}(\forall i=1,2, \ldots, n)$. One set of $\left\{b_{i}\right\}$ is called an individual of the population in a generation in GA terminology. The coefficients $\left\{b_{i}\right\}$ are assumed to be real numbers without losing generality. Each $b_{i}$ is coded into a binary representation with length $L$ via the mapping formula

$$
b_{i}=x_{\min }+\frac{x_{\max }-x_{\min }}{2^{L}-1} \sum_{j=0}^{L-1} b_{L-j}^{(\mathrm{B})} 2^{j}
$$

for $x_{\min } \leqslant b_{i} \leqslant x_{\max }$. The superscripted notation $b_{L-j}^{(\mathrm{B})}$ (for $j=0,2, \ldots, L-1)$ is the $(L-j)$ th digit in the binary representation of $b_{i}$ when one counts the digits from left to right. In other words, $2^{L}$ numbers out of an infinitely many real numbers defined in the domain $\left[x_{\min }, x_{\max }\right]$ are represented in our binary expression. For example, $x_{\min }\left(x_{\max }\right)$ is equal to 000000000000000 (111111111111111) in the binary form. With the binary representation, one individual is characterized by $n$ length- $L$ binary-bit strings. The whole string (of size $n \times L$ ) is sometimes called a chromosome and the segments on it are called genes (with size $L$ ). The minimum value, the maximum value, and the gene length $L$ should be chosen large enough in order to cover the range in which one can reproduce the optimal solution and reach the wanted precision. One important note is that the retrieved phase should agree with the true phase up to an arbitrary constant phase because we are fitting the trace/autocorrelation and they are the same with any extra constant phase added. In principle, as long as $x_{\min }$ and $x_{\max }$ are far part enough, we should be able to find the solution.

The evolution operations (such as crossover and mutation) act on this binary representation, i.e., $b_{L-j}^{(\mathrm{B})}$ (for $j=$ $0,2, \ldots, L-1)$. The real-number expression and the binary expression can be interchanged to each other via Eq. (A1) and its inverted version.

After setting up the problem, we initialize the population. Assume there are $N$ individuals in one generation (note that $N$ is different from $n$ ). A uniform random real number generator (between $[0,1]$ ) is used to produce the population in the first generation. If the random number is less then 0.5 , then the digit is set to zero. Otherwise, it is set to one. A total number of $(N \times n \times L)$ random numbers are thrown for initialization.

Next, we evaluate the fitness of each individual. The fitness function $F$ is defined, closely related to Eq. (8), as

$$
F\left[\left\{b_{i}\right\}\right]=-E\left[\left\{b_{i}\right\}\right],
$$

where the negative sign is added to ensure a larger value of $F$ corresponds to a better fitness. The fitness level of an individual is used to perform the third step: selection of parents. We use tournament selection. Two individuals of the population are selected out at random and the one with greater fitness is chosen as the first parent. The same selection scheme is applied again to select the second parent. Next, the selected pairs then go through crossover to produce one new offspring. The crossover scheme applied here is uniform crossover with a crossover probability $P_{\text {cross }}=0.5$. This means that every bit of the offspring is independently chosen from the two parents with equal probability. A different crossover probability could be adopted if one wishes the offspring to inherit more genetic information from one parent than the other. Other crossover schemes exist as well [21] - for example, a single-point crossover and its variant, the $M$-point crossover $(M>1)$. However, the uniform crossover avoids the potential bias for two neighboring bits to be inherited together, so we prefer it in our calculations.

This selection and crossover scheme are performed $N$ times such that $N$ offspring are produced. Nevertheless, the offspring produced at this stage are not finalized yet. We allow genetic variations, which are not inherited from parents, to maintain genetic diversity and prevent premature convergence of the solution. The mutation operator is often used to achieve this goal. Various mutation schemes exist-for example, jump mutation and creep mutation. In jump mutation, we go through all bits in the population and flip the bit when a drawn random real number is less than the mutation probability, $P_{\text {mutate }}$. We typically set $P_{\text {mutate }}=1 / N$. For creep mutation, we need to throw another random real number. If it is smaller than the creep probability, then we perform the creep mutation. Creep mutation, as the name suggests, mutates in the near area of the parents. Once creep mutation is activated, the individual is increased or decreased to the nearest possible real numbers in the parameter space with equal probability. $P_{\text {creep }}$ should be not too large. We typically set $P_{\text {creep }}=0.02-0.04$ for $N=20-100$. These two mutation schemes can be used together or independently. After mutation, a new generation is formed. If one wants to keep the best individual from the previous generation, elitism can be applied. That is, if the best individual is not reproduced, we randomly replace one individual by the best one.

Instead of using mutation, a so-called "micro-GA" may be used to replace mutation after the new generation is produced. What the micro-GA does is the following: first we check whether the population is "converged." We measure the number of different bits from the best member. If the total number of different bits are less than $0.05 \times[(N-1) \times n \times$ $L]$, then the population is considered "converged." If so, we restart the next run by using a new generation formed by the best individual and the new randomly generated parents. The whole procedure is repeated until we meet the requirement of termination.

The efficiency of the GA depends on many factors. Experimenting on the choice of the input parameters for the GA is necessary. Apart from selections of the input parameters, one intrinsic question one should always ask is how good the $B$-spline basis functions are to describe the true phase. Our strategy to overcome the problem is to run over different combinations of $(n, k)$ for a few generations (for example, $100)$ and pick the basis set with fast descent in $E\left[\left\{b_{i}\right\}\right]$. Based on our observations, running $k$ from 4-7 and $n$ from 10-14 would often guide us to select the best $n$ and $k$ for our cases. One last freedom in the $B$-spline basis function is how one places the $(n+k)$ knots in the energy regime of interest. Our experiences show that placing more knots near the central energy of the XUV pulse always allows a faster evolution of the GA to the optimal solution. 
[1] P. M. Paul, E. S. Toma, P. Breger, G. Mullot, F. Augé, P. Balcou, H. G. Muller, and P. Agostini, Science 292, 1689 (2001).

[2] M. Hentschel et al., Nature (London) 414, 509 (2001).

[3] C. D. Lin, A. T. Le, C. Jin, and H. Wei, Attosecond and Strong-Field Physics Principles and Applications (Cambridge University Press, 2018).

[4] G. Sansone et al., Science 314, 443 (2006).

[5] E. Goulielmakis et al., Science 320, 1614 (2008).

[6] K. Zhao et al., Opt. Lett. 37, 3891 (2012).

[7] J. Li, X. Ren, Y. Yin, K. Zhao, A. Chew, Y. Cheng, E. Cunningham, Y. Wang, S. Hu, Y. Wu, M. Chini, and Z. Chang, Nat. Commun. 8, 186 (2017).

[8] T. Gaumnitz, A. Jain, Y. Pertot, M. Huppert, I. Jordan, F. A.Lamas, and H. J. Wörner, Opt. Express 25, 27506 (2017).

[9] H. G. Muller, Appl. Phys. B 74, S17 (2002).

[10] Y. Mairesse and F. Quéré, Phys. Rev. A 71, 011401 (2005).

[11] M. Chini, S. Gilbertson, S. D. Khan, and Z. Chang, Opt. Express 18, 13006 (2010).
[12] X. Zhao, Hui Wei, Yan Wu, and C. D. Lin, Phys. Rev. A 95, 043407 (2017).

[13] P. D. Keathley, S. Bhardwaj, J. Moses, G. Laurent, and F. X. Kärtner, New J. Phys. 18, 073009 (2016).

[14] X. Zhao, Hui Wei, W.-W. Yu, and C. D. Lin, Phys. Rev. A 98, 053404 (2018).

[15] X. Zhao, Hui Wei, C.-L. Wei, and C. D. Lin, J. Opt. 19, 114009 (2017).

[16] J. Gagnon, E. Goulielmakis, and V. S. Yakovlev, Appl. Phys. B 92, 25 (2008).

[17] X. M. Tong and C. D. Lin, J. Phys. B: At. Mol. Opt. Phys. 38, 2593 (2005).

[18] H. Bachau, E. Cormier, P. Decleva, J. E. Hansen, and F. Martín, Rep. Prog. Phys. 64, 1815 (2001).

[19] M. Schultze et al., Science 328, 1658 (2010).

[20] H. Wei, T. Morishita, and C. D. Lin, Phys. Rev. A 93, 053412 (2016).

[21] O. Hasançebi and F. Erbatur, Comput. Struct. 78, 435 (2000). 